CASE REPORT

\author{
R.D. Welling
}

J.A. Olson, Jr.

P.G. Kranz

J.D. Eastwood

J.K. Hoang

\section{Bilateral Retropharyngeal Parathyroid Hyperplasia Detected with 4D Multidetector Row CT}

SUMMARY: We present a case of bilateral retropharyngeal parathyroid hyperplasia detected with 4D-CT in a patient with persistent primary hyperparathyroidism and failed neck exploration. We discuss the embryologic basis of ectopic retropharyngeal parathyroid adenomas and hyperplasia and the utility of $4 \mathrm{D}-\mathrm{CT}$ in their localization for surgical planning.

ABBREVIATIONS: PTH = parathyroid hormone; SPECT = single-photon emission computed tomography
$\mathbf{P}$ rimary hyperparathyroidism is caused by a single-gland adenoma in most patients $(\leq 90 \%)$ or multiglandular hyperplasia $(10 \%-15 \%){ }^{1}$ Ectopic parathyroid adenomas and hyperplasia, defined by their location in sites other than the juxtathyroid position, are a well-documented cause of primary hyperparathyroidism and are reported in $\leq 22 \%$ of cases. $^{2,3}$ When an adenoma or glandular hyperplasia is ectopic, findings of the initial work-up with sonography and sestamibi scintigraphy may be negative. In such cases, MR imaging and CT are often performed. These second-line investigations have become increasingly important since the surgical treatment of parathyroid masses has transitioned from bilateral 4-gland exploration to unilateral minimally invasive parathyroidectomy. ${ }^{4,5}$ The need to accurately localize parathyroid lesions and advances in CT technology have led to new protocols such as $4 \mathrm{D}-\mathrm{CT}$. $^{6-8}$

We present a case of ectopic bilateral retropharyngeal parathyroid hyperplasia detected on $4 \mathrm{D}-\mathrm{CT}$ and review its role and technique.

\section{Case Report}

A 47-year-old woman was referred to our institution for evaluation of persistent primary hyperparathyroidism after bilateral neck exploration 4 years prior. At that time, she had undergone right inferior parathyroidectomy and biopsy of the inferior left parathyroid gland, which revealed hyperplasia. The superior parathyroid glands were not identified.

Postoperatively, the patient continued to experience hypercalcemia and was treated with the calcimimetic, cinacalcet (Sensipar). Despite medical therapy, the patient's serum PTH and serum and urine calcium levels remained elevated. She continued to have symptoms of fatigue, memory loss, and muscle aches and had osteopenia detected by a dual energy x-ray absorptiometry scan. Given her biochemical abnormalities, symptoms, and bone loss, she again presented for surgical evaluation.

The imaging work-up began with sonography, which did not demonstrate a parathyroid adenoma in the usual juxtathyroid locations. Next she underwent sestamibi imaging with SPECT correlation (Fig 1). Two-hour planar sestamibi imaging demonstrated minimal

Received February 5, 2010; accepted after revision February 20.

From the Departments of Radiology (R.D.W., P.G.K., J.D.E., J.K.H.) and Surgery (J.A.O.), Division of General Surgery, Duke University Medical Center, Durham, North Carolina.

Please address correspondence to R.D. Welling, MD, Department of Radiology, Box 3808, Erwin Rd, Duke University Medical Center, Durham, NC 27710; e-mail: rodney.welling@ duke.edu

DOI 10.3174/ajnr.A2104 residual uptake in the right neck inferior to the submandibular gland. Fused SPECT imaging demonstrated a region of increased radiotracer activity in the right retropharyngeal space, superior to the upper pole of the right thyroid. No activity was present in the left neck on delayed sestamibi images.

Finally, 4D-CT of the neck and mediastinum was performed (Fig 2). Our protocol involves imaging with 64-section multidetector row CT after $120 \mathrm{~mL}$ of iopamidol (Isovue-300; Bristol-Meyers Squibb, Princeton, New Jersey) is administered intravenously via an 18-ga intravenous cannula in the right antecubital fossa at a rate of $3 \mathrm{~mL} / \mathrm{s}$, followed by a $50-\mathrm{mL}$ saline flush. We acquire contiguous axial images from the lower margin of the mandible to the level of the inferior aortic arch in arterial (25-second delay) and venous phases (80-second delay) with the following settings: 0.625 section thickness; gantry rotation time, 0.8 seconds; $20-\mathrm{cm}$ FOV; $120 \mathrm{kV}$ (peak); and automatic tube current modulation (Smart mA; GE Healthcare, Milwaukee, Wisconsin). Reformatted images in the 2 phases are sent to a PACS as 2.5-mm-thick contiguous images in the axial, coronal, and sagittal planes.

On arterial phase images, 2 hyperenhancing lesions, right larger than left, were seen in the retropharyngeal space at the level of the pyriform sinus. On delayed images, there was rapid washout of contrast in the lesions, which were both oval on reformatted sagittal and coronal images.

On the basis of these findings, the surgeon performed a targeted neck exploration in the right retropharyngeal space through a $3-\mathrm{cm}$ incision and identified a 600-mg parathyroid mass. After its removal, intraoperative serum PTH dropped from its highest baseline level but remained elevated. Because of this plateau of the intraoperative PTH level and a probable second abnormality on $4 \mathrm{D}-\mathrm{CT}$, the surgeon explored the left retropharyngeal space and found a second 360-mg mass. Following resection of the second mass, the intraoperative serum PTH level normalized and the procedure was concluded.

Pathology showed that both parathyroid masses were hypercellular, consistent with multiple gland parathyroid hyperplasia. At 2-week and 1-month follow-ups, the total calcium and PTH levels were normal.

\section{Discussion}

Parathyroid adenomas and hyperplasia are most commonly found posterior to the thyroid between the hyoid and sternal notch. Ectopic locations for parathyroid tumors include mediastinal, intrathyroid, intracarotid sheath, and intraneural sites. $^{3,9}$ Retropharyngeal ectopic parathyroid lesions are rare and, when present, are a diagnostic challenge for the radiolo- 

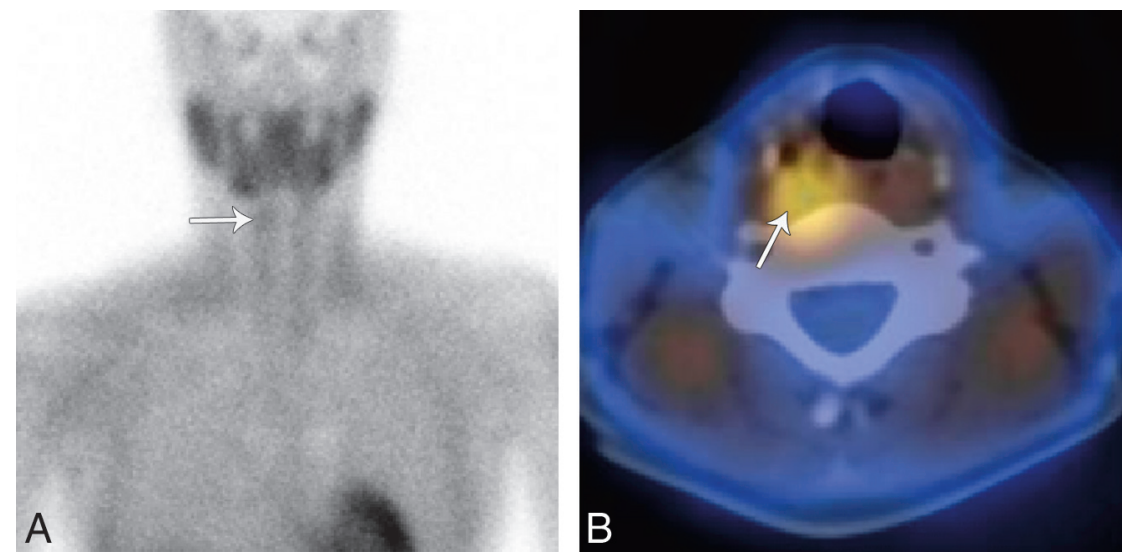

Fig 1. Technetium Tc99m sestamibi imaging with SPECT. A, A 2-hour planar sestamibi image shows minimal residual uptake in the right neck (arrow) inferior to the submandibular gland. $B$, A fused axial SPECT image demonstrates tracer activity (arrow) superior and posterior to the right thyroid lobe in the region of the right retropharyngeal space.
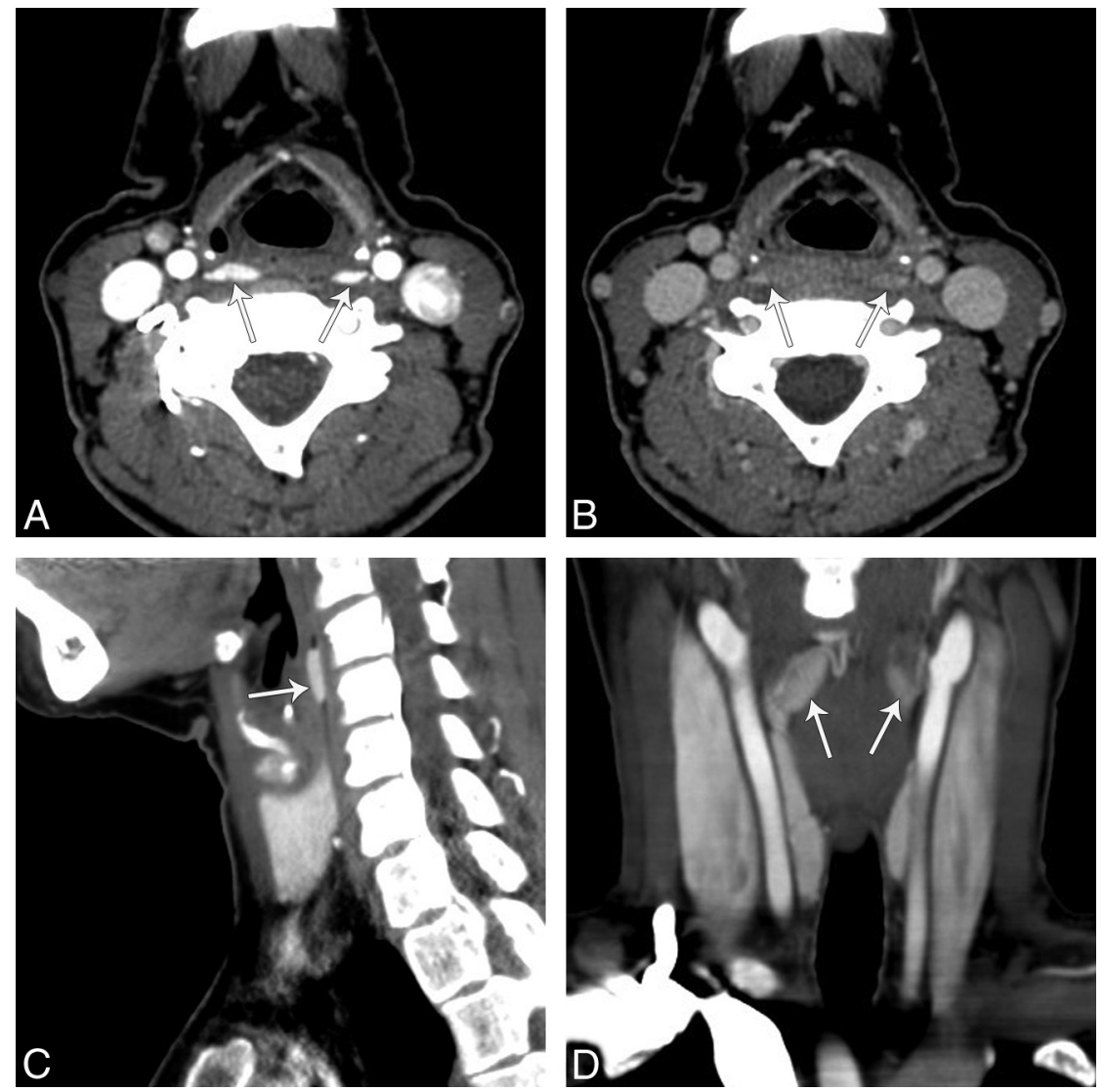

Fig 2. 4D-CT of the neck showing bilateral retropharyngeal masses. $A$, An arterial phase axial image of the neck at the level of the pyriform sinus demonstrates 2 hyperenhancing lesions (arrows), right larger than left, in the retropharyngeal space. $B, 0$ ndelayed images at the same level there was rapid washout of contrast in the lesions. Reformatted right sagittal $(C)$ and coronal arterial phase $(D)$ images demonstrate that hyperenhancing lesions (arrows) in the retropharyngeal space have an oval shape.

gist and surgeon. A case of bilateral ectopic retropharyngeal parathyroid hyperplasia has not previously been reported.

The retropharyngeal location of the parathyroid gland is due to the common embryologic origin of the superior parathyroid gland and the apex of the pyriform sinus from the fourth branchial pouch of the fetal pharyngeal wall. ${ }^{10}$ If the parathyroid gland fails to separate from the pyriform sinus, the structures may migrate together to a retropharyngeal location. Hence, retropharyngeal parathyroid tumors are typically found at the level of the pyriform sinus, as shown in our patient.
Bilateral retropharyngeal parathyroid tumors could be mistaken for venous plexuses on a routine axial enhanced CT scan (Fig 2B). 4D-CT helped us make a correct diagnosis. The original description of $4 \mathrm{D}$-CT includes image sets in 3 planes (axial, coronal, sagittal). ${ }^{6}$ The "fourth" dimension of $4 \mathrm{D}-\mathrm{CT}$ is the perfusion information derived from noncontrast, arterial, and delayed (venous) phase imaging. In this reported case, the arterial phase imaging was useful for detection of discrete hyperenhancing tumors, and delayed images showed rapid washout of contrast, distinguishing the lesions from surrounding venous structures (Fig 2). The noncontrast phase 
has been removed from our protocol because we did not find that it helped with diagnosis.

Rodgers et $\mathrm{al}^{6}$ reported that $4 \mathrm{D}-\mathrm{CT}$ had improved sensitivity $(88 \%)$ over sestamibi imaging $(65 \%)$ and sonography (57\%) when the studies were used to lateralize hyperfunctioning parathyroid glands to 1 side of the neck. Furthermore, when used to localize parathyroid tumors to the correct quadrant of the neck, the sensitivity of 4D-CT (70\%) was significantly higher than that of sestamibi imaging (33\%) and sonography $(29 \%)$. In another study of patients with repeat neck surgery, 4D-CT more often localized and lateralized hyperfunctioning parathyroid tissue than sestamibi imaging. ${ }^{8}$ $4 \mathrm{D}-\mathrm{CT}$ has also been shown to have better sensitivity for the detection of multiglandular disease, which is a common cause for repeat surgery. ${ }^{6}$ The advantage of $4 \mathrm{D}$-CT was illustrated in our case because the sestamibi study did not demonstrate the surgically proved left retropharyngeal hyperplastic gland, a phenomenon thought to be due to rapid washout of radiotracer from the left parathyroid tumor, ${ }^{11,12}$ a potential pitfall of sestamibi imaging.

A disadvantage of $4 \mathrm{D}-\mathrm{CT}$ is its increased radiation exposure to the patient. To reduce the radiation dose, we modified our current protocol to exclude the precontrast imaging described in the original studies of $4 \mathrm{D}-\mathrm{CT}$. We also use dosereduction techniques of automatic tube current modulation, and we limit the superior level of imaging to the lower mandible to exclude the lens of the orbit and the brain. Despite these attempts, the dose-length product for arterial and venous phase in this study was $1300 \mathrm{mGy} \mathrm{cm}$, which is equivalent to just $<2$ CT neck scans with an optimized dose-reduction technique. ${ }^{13}$ Hence, 4D-CT should be reserved for cases of primary hyperparathyroidism with indeterminate or negative findings on sonography and sestamibi scintigraphy.

MR imaging with contrast is another imaging option, depending on the experience of the institution. ${ }^{14,15} \mathrm{MR}$ imaging has a sensitivity similar to that of sonography and sestamibi imaging. ${ }^{16}$ On MR imaging, parathyroid adenomas may have variable T1 and T2 signal-intensity patterns. Contrast imaging increases the sensitivity for detection of lesions that are $\mathrm{T} 1$ and $\mathrm{T} 2$ isointense, but false-negative results can still arise from motion artifacts or because adenomas may have signal-intensity and enhancement characteristics similar to those of cervical lymph nodes. ${ }^{17}$

This case of bilateral retropharyngeal parathyroid hyperplasia shows the value of $4 \mathrm{D}-\mathrm{CT}$ in defining a discrete para- thyroid lesion and in determining the enhancement characteristics of the lesion. Accurate localization of parathyroid tumors is important for repeat parathyroid surgery and for minimally invasive directed parathyroidectomy.

\section{Acknowledgment}

We thank Michael Hanson, MD, for his clinical input.

\section{References}

1. Schachter PP, Ayesh S, Schneider T, et al. Expression of kinase genes in primary hyperparathyroidism: adenoma versus hyperplastic parathyroid tissue. Surgery 2002;132:1094-98, discussion 1098-99

2. Thompson NW, Eckhauser FE, Harness JK. The anatomy of primary hyperparathyroidism. Surgery 1982;92:814-21

3. Lumachi F, Zucchetta P, Varotto S, et al. Noninvasive localization procedures in ectopic hyperfunctioning parathyroid tumors. Endocr Relat Cancer 1999;6:123-25

4. Ruda JM, Hollenbeak CS, Stack BC Jr. A systematic review of the diagnosis and treatment of primary hyperparathyroidism from 1995 to 2003. Otolaryngol Head Neck Surg 2005;132:359-72

5. Adil E, Adil T, Fedok F, et al. Minimally invasive radioguided parathyroidectomy performed for primary hyperparathyroidism. Otolaryngol Head Neck Surg 2009;141:34-38

6. Rodgers SE, Hunter GJ, Hamberg LM, et al. Improved preoperative planning for directed parathyroidectomy with 4-dimensional computed tomography. Surgery 2006;140:932-40, discussion 940-41

7. Philip M, Guerrero MA, Evans DB, et al. Efficacy of 4D-CT preoperative localization in 2 patients with MEN 2A. J Surg Educ 2008;65:182-85

8. Mortenson MM, Evans DB, Lee JE, et al. Parathyroid exploration in the reoperative neck: improved preoperative localization with $4 \mathrm{D}$-computed tomography. J Am Coll Surg 2008;206:888-95, discussion 895-96

9. Chan TJ, Libutti SK, McCart JA, et al. Persistent primary hyperparathyroidism caused by adenomas identified in pharyngeal or adjacent structures. World J Surg 2003;27:675-79

10. Benson MT, Dalen K, Mancuso AA, et al. Congenital anomalies of the branchial apparatus: embryology and pathologic anatomy. Radiographics 1992;12:943-60

11. Benard F, Lefebvre B, Beuvon F, et al. Rapid washout of technetium-99m-MIBI from a large parathyroid adenoma. J Nucl Med 1995;36:241-43

12. Nguyen BD. Parathyroid imaging with Tc-99m sestamibi planar and SPECT scintigraphy. Radiographics 1999;19:601-14, discussion 615-16

13. Russell MT, Fink JR, Rebeles F, et al. Balancing radiation dose and image quality: clinical applications of neck volume CT. AJNR Am J Neuroradiol 2008;29:727-31

14. Fakhran S, Branstetter BF 4th, Pryma DA. Parathyroid imaging. Neuroimaging Clin N Am 2008;18:537-49, ix

15. Lee VS, Spritzer CE, Coleman RE, et al. The complementary roles of fast spinecho MR imaging and double-phase $99 \mathrm{~m}$ Tc-sestamibi scintigraphy for localization of hyperfunctioning parathyroid glands. AJR Am J Roentgenol 1996; 167:1555-62

16. Lopez Hanninen E, Vogl TJ, Steinmuller T, et al. Preoperative contrastenhanced MRI of the parathyroid glands in hyperparathyroidism. Invest Radiol 2000;35:426-30

17. Johnson NA, Tublin ME, Ogilvie JB. Parathyroid imaging: technique and role in the preoperative evaluation of primary hyperparathyroidism. AJR Am J Roentgenol 2007;188:1706-15 